\title{
Sequence Stratigraphy and Sedimentary Facies of Feixianguan Formation in the Kaijiang-Liangping Area of Sichuan Basin, China
}

\author{
Qingbin Xie1,2, Zhifang Wang2, Yongli Ouyang3, Miaoyi Zhang², Bing Liu², Lvli Wang2, \\ Xiaojing Liu ${ }^{2}$ \\ ${ }^{1}$ Faculty of Petroleum, China University of Petroleum-Beijing at Karamay, Beijing, China \\ ${ }^{2}$ College of Geosciences, China University of Petroleum (Beijing), Beijing, China \\ ${ }^{3}$ PetroChina Research Institute of Petroleum Exploration \& Development-Langfang Branch, Langfang, China \\ Email: xieqingbin@cup.edu.cn
}

How to cite this paper: Xie, Q.B., Wang, Z.F., Ouyang, Y.L., Zhang, M.Y., Liu, B., Wang, L.L. and Liu, X.J. (2020) Sequence Stratigraphy and Sedimentary Facies of Feixianguan Formation in the Kaijiang-Liangping Area of Sichuan Basin, China. Open Journal of Geology, 10, 641-660.

https://doi.org/10.4236/ojg.2020.106029

Received: March 12, 2019

Accepted: June 20, 2020

Published: June 23, 2020

Copyright $\odot 2020$ by author(s) and Scientific Research Publishing Inc. This work is licensed under the Creative Commons Attribution International License (CC BY 4.0).

http://creativecommons.org/licenses/by/4.0/

\begin{abstract}
The Feixianguan formation in the Kaijiang-Liangping basin has been the focus of extensive research on multiple aspects. Based on field survey, core observation, laboratory analysis and seismic data interpretation, the sequence stratigraphy and sedimentary facies of the Kaijiang-Liangping area are studied. Four sequence boundaries and three maximum flooding surfaces of the Feixianguan formation are detected in this area. Three third-order sequences are identified as first sequence (SQ1), the second sequence (SQ2), and the third sequence (SQ3) in which SQ1 corresponds to the member 1 of the Feixianguan formation, SQ2 corresponds to the member 2, and SQ3 corresponds to the member 3 and member 4 . Member 1,2, 3 and 4 are lithologic sections divided by predecessors. On the basis of this sequence division and their sedimentary marks, the facies of the Feixianguan formation can be divided into open platform and evaporate platform categories. The open platform is composed of three subfacies identified as platform bank or basin marginal bank, interbank, and platform basin. Thus, a sedimentary evolution model is established. According to the sedimentary and seismic characteristics of the Kaijiang-Liangping area, we determine that two oolitic bank models, the aggradation model and the progradation model, are developed in this area. The aggradation model is developed in the Longgang region, which includes the basin marginal bank as a favorable exploring zone. The progradation model is developed in the Jiulongshan and Longhui areas, besides the basin marginal bank, the favorable exploration zones also include the oolitic bank developing areas of the inner basin.
\end{abstract}




\section{Keywords}

Sichuan Basin, Kaijiang-Liangping Area, Feixianguan Formation, Sedimentary Facies

\section{Introduction}

The concept Kaijiang-Liangping trough was put forward in the end of the 20th century [1]. Since then numerous studies have been conducted on the sequence and sedimentary facies of the Kaijiang-Liangping Feixianguan Formation [2]-[7]. Thus far, two different perspectives have been reported on the division of the stratigraphic sequence of the Feixianguan Formation: two third-order sequences and three third-order sequences. Previous research on the sedimentary facies also shows different conclusions. Yang and Wen determined that the facies of the Kaijiang-Liangping Feixianguan Formation evolved from the trough to the continental shelf [2]. Wei suggested that the facies gradually evolved from the basin to the slopes, platform margin bank, open platform, restricted platform, and evaporation platform [3]. Ma deemed that the facies of the Kaijiang-Liangping area from the Changxing period to the Early Triassic period were produced in an environment of platform shelf in relatively deep waters of a carbonate platform [4]. In addition to these inconsistencies, little research has been conducted on the inner basin.

In the present study, the sequence stratigraphy of the sedimentary facies in the outcrop is examined, and laboratory tests, drilling and logging information, and seismic activity are analyzed. The results indicate that the sedimentary model of the early Triassic Feixianguan period, northeast Sichuan, is a shallow sea carbonate platform. This study is based on the sequence division in the Kaijiang-Liangping basin area. The objectives include examining the sedimentary facies of the Feixianguan Formation in the Kaijiang-Liangping area, building a sedimentary evolution model, and putting forward two development models for an oolitic beach involving progradation and aggradation, respectively. It is hoped that the results will provide a geologic basis for further exploration in the Kaijiang-Liangping basin.

\section{Geological Background}

The Kaijiang-Liangping basin, Northeast Sichuan, with an area approximately 2 $\times 10^{4} \mathrm{~km}^{2}$, geographically covers the cities of Guangyuan, Nanchong, Bazhong, and Dazhou in Sichuan. The eastern border is a fault boundary extending to the Nanjiang, Tongjiang, Xuanhan, Kaixian area; the western border is an onlap boundary stretching to the Jiangge, Cangxi, Yilong, Dazhu area; the north borders the Guangyuan-Wangcang trough; and the south border crosses Liangping, Wanzhou, and Chongqing (Figure 1).

The tectonic and sedimentary evolution of the Feixianguan Formation in 
northeast Sichuan is directly related to expansion and shrinkage of the South Qinling ocean, which resulted in the formation of the Kaijiang-Liangping basin [8]. On the platform, the underlying stratum of the Feixianguan Formation is the Changxing Formation; a depositional hiatus exists between them. In the basin, the underlying stratum is the Dalong Formation, with successive sedimentation occurring between them. The Feixianguan Formation is divided into four members. The member 1 comprises dark gray medium-bed limestone and marl; the member 2 is gray marl with thin bio-clastic micrite; the member 3 comprises light gray medium-bed micrite, oolitic limestone, and calcarenite; and the member 4 comprises regional purple shale, thin marl, and thin dolomicrite with gypsum.

\section{Characteristics of Sequence Stratigraphy}

The key to sequence classification is the recognition of sequence boundaries. A sequence boundary is defined as an unconformity or its correlative conformity [9]. In this study, four sequence boundaries and three maximum flooding surfaces were identified in the Feixianguan Formation in the study area (Figure 2): SB1, SB2, SB3, and SB4 and MFS1, MFS2, and MFS3, respectively. SB1 is the interface of the Changxing Formation or Longtan Formation, according to the conformity surface, and the Feixianguan Formation; SB2 and SB3 are the bottom interfaces of the member 2 and the member 3 of the Feixianguan Formation, respectively; and SB4 is the interface of the member 4 of the Feixianguan Formation and the Jialingjiang Formation. The features of each interface are given in Table 1.

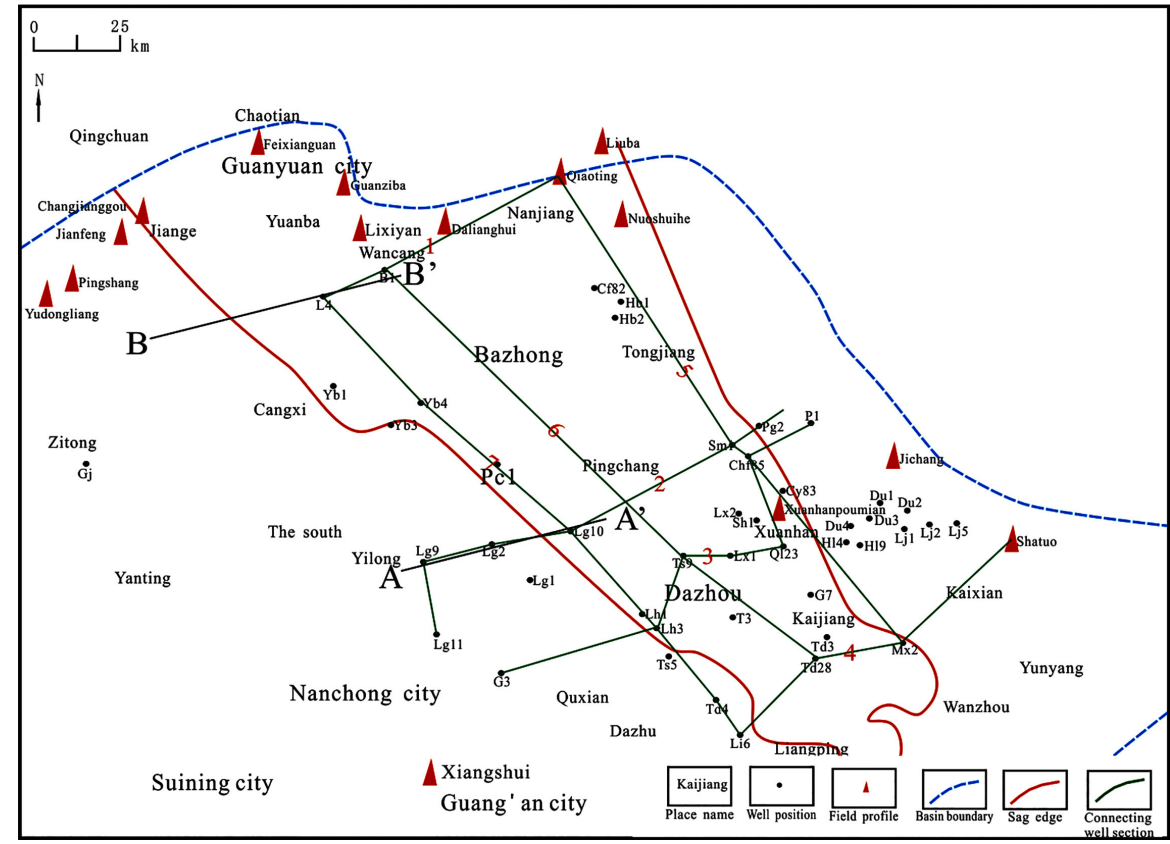

Figure 1. Position and boundaries of the Kaijiang-Liangping basin in the northeast Sichuan. 


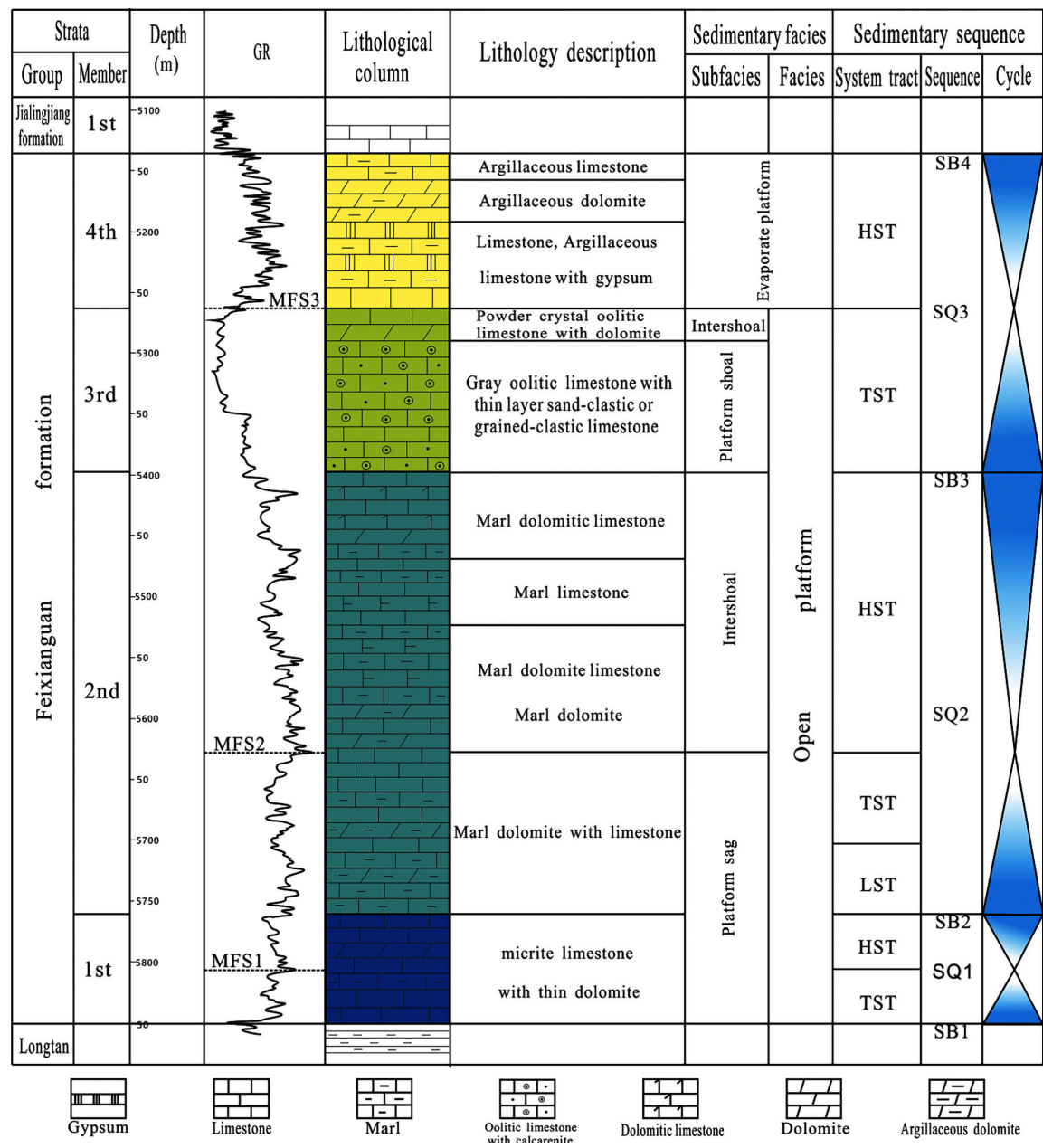

Figure 2. Synthesis column section of well Long4 platform basin in the Kaijiang-Liangping area.

Table 1. Sequence boundary features of the Feixianguan Formation in the Kaijiang-Liangping area.

\begin{tabular}{|c|c|c|c|c|c|}
\hline \multirow{2}{*}{ Interface } & \multirow{2}{*}{ strata } & \multicolumn{4}{|l|}{ identifying characteristics } \\
\hline & & lithology & electrical property & seismic reflection & Palaeobios \\
\hline \multirow[b]{2}{*}{ SB4 } & Jialingjiang Fm & $\begin{array}{l}\text { Medium to thin layer } \\
\text { micrite }\end{array}$ & $\begin{array}{l}\text { Relative low GR, } \\
\text { high Rt }\end{array}$ & & $\begin{array}{l}\text { tirolites-prolarmite } \\
\text { Owenites-meekoceras }\end{array}$ \\
\hline & top of Feixianguan Fm & $\begin{array}{l}\text { gypseous limestone, } \\
\text { gypsum and Purple } \\
\text { clayshale }\end{array}$ & high GR, low Rt & downlap & $\begin{array}{l}\text { Claraia rich of Eumorphotis } \\
\text { multiformis Flemingitsekoninckites, } \\
\text { Ophiceras }\end{array}$ \\
\hline \multirow[b]{2}{*}{ SB3 } & bottom of the member & micrise, marl & high GR, low Rt & onlap & \\
\hline & top of the member 2 & $\begin{array}{l}\text { oolitic limestone, } \\
\text { micrite limestone }\end{array}$ & low GR, high Rt & toplap or truncation & \\
\hline \multirow{2}{*}{ SB2 } & bottom of the member 2 & mudstone & high GR, low Rt & onlap or downlap & \\
\hline & top of the member 1 & limestone & low GR, high Rt & & \\
\hline \multirow[b]{2}{*}{ SB1 } & $\begin{array}{l}\text { bottom of Feixiangan } \\
\text { Fm }\end{array}$ & marlstone, limestone & high GR, low Rt & $\begin{array}{l}\text { moderate reflection, } \\
\text { onlap }\end{array}$ & $\begin{array}{l}\text { Hindeodus parvus, Otoceras and } \\
\text { Claraiawangi }\end{array}$ \\
\hline & $\begin{array}{l}\text { Changxing Fm } \\
\text { (Dalong Fm) }\end{array}$ & $\begin{array}{l}\text { reef limestone, dolomitic } \\
\text { limestone, siliceous rock } \\
\text { and carbonaceous shale }\end{array}$ & low GR, high Rt & & $\begin{array}{l}\text { fusulinides, rugose corals, tabulate } \\
\text { corals, trilobites hyolithids }\end{array}$ \\
\hline
\end{tabular}


SB1 is the interface of the Changxing Formation and the Feixianguan Formation and is also an important lithology trans-Formation interface. The lithology turns from thick, massive chert limestone and reef limestone at the top of the Changxing Formation, or siliceous rock and carbonaceous shale in the Dalong Formation, into thin layers of argillaceous limestone and micrite limestone of the Feixianguan Formation (Figure 3). The electrical behavior turns from low gamma ray (GR) and high resistivity of the true formation (Rt) into high GR and low Rt. Moreover, the Permian extinction led to obvious decreases in species in the Feixianguan period, particularly in Hindeodus parvus, Otoceras, and Claraiawangi; this biological mutation phenomenon is indicated by the fossil presence at the boundary.

The seismic reflection characteristics of each interface are obvious (Figure 4). The SB1 shows moderate reflection characteristics and obvious onlap phenomena at the boundaries of the basin. SB2 is the interface of the member 1 and member 2 of the Feixianguan Formation, which is often characterized by mudstone overlapping the limestone and by onlap at the bottom and truncation at the top of the member 2 of the Feixianguan Formation. SB3 is the interface of the member 2 and member 3 of the Feixianguan Formation, with onlap evident at the bottom of the member 3 of the Feixianguan Formation. This interface is also an important because it shows mutations in lithology and electrical behavior (Figure 5). SB4, the interface of the Feixianguan and Jialingjiang Formations (Figure 6(a)), shows strong reflection characteristics. The lithology is mainly tan or fuchsia mud stone (Figure 6(b)) and shale dolomite intercalated with gypsum rock. Typical desiccation cracks (Figure 6(c)) and bird's eye structures caused by exposure and flaggy micrite formed by transgression are present in the Jialingjiang Formation. The main paleontology in the Feixianguan Formation includes Claraia and abundant combinations of Eumorphotis multiformis, Flemingites, Koninckites, andOphiceras. The Jialingjiang Formation is rich in combinations of Tirolites-Prolarnites and Owenites-Meekoceras. The features of the interfaces between the two formations are obvious.

According to the recognition features of sequence boundaries in the study area, the Feixianguan Formation was divided into three third-order sequences in the Kaijiang-Liangping area including SQ1, which corresponds to the member 1 of the Feixianguan Formation; SQ2, which corresponds to the member 2; and SQ3, which corresponds to the member 3 and member 4 . Both the transgressive systems tract (TST) and high-stand systems tract (HST) are developed in SQ1, SQ2, and SQ3 on the platform. In the basin, TST and HST are developed in SQ1 and SQ3; low-stand system tract (LST), TST and HST are developed in SQ2 (Figure 7).

\section{Characteristics of Sedimentary Facies and Sedimentary Evolution Model}

\subsection{Types and Characteristics of Sedimentary Facies}

The types and characteristics of the sedimentary facies of the Feixianguan 

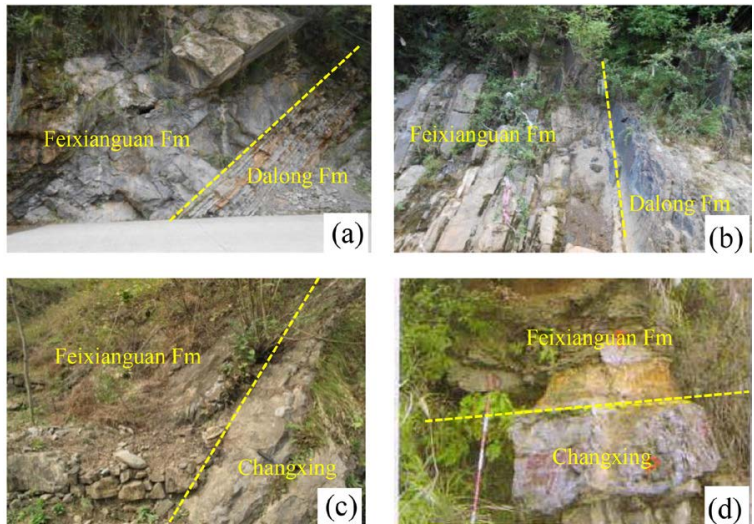

Figure 3. SB1 of the Feixianguan Formation and the Changxing (Dalong) Formation. (a) interface of the Feixianguan Formation and the Dalong Formation of the Dalianghui section in Wancang; (b) interface of the Feixianguan Formation and the Dalong Formation of the Jianfeng section; (c) interface of the Feixianguan Formation and the Changxing Formation of the Wangjiagou section in Wancang; (d) interface of the Feixianguan Formation and the Changxing Formation of the Chen'erwan section in Huaying Mountain.

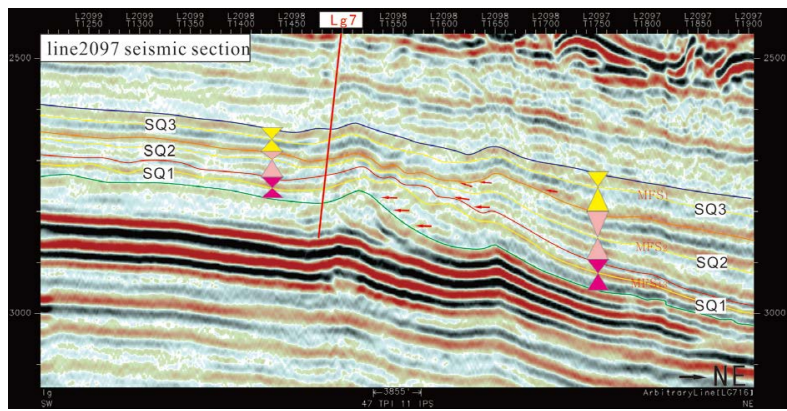

Figure 4. Characteristics of SB1, SB2, SB3, and SB4 of line 2097 on the seismic section.
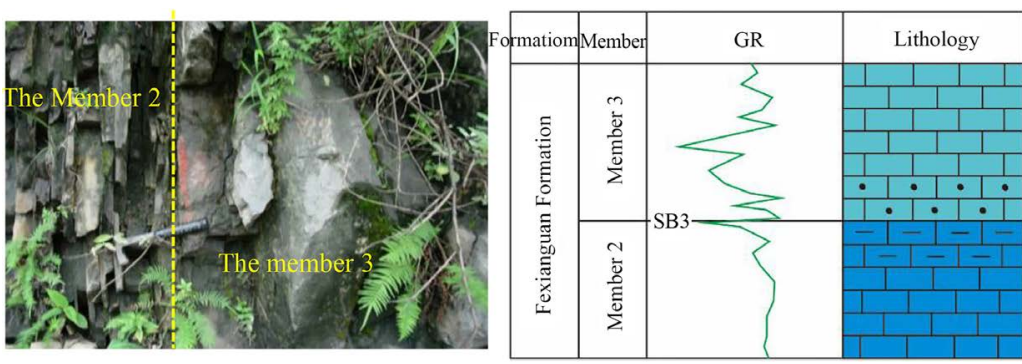

Figure 5. Lithology and electrical properties of the Nanjiang-Qiaoting section and well Bian2.
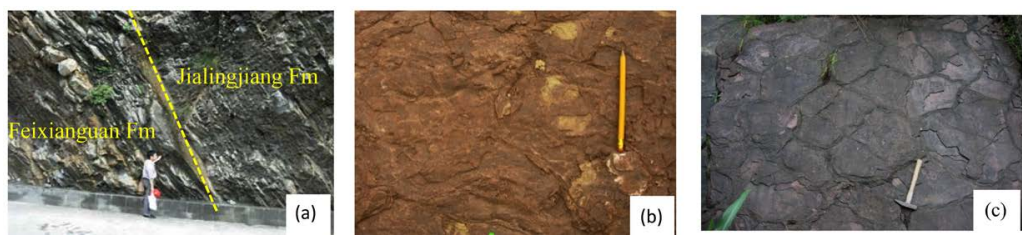

Figure 6. Characteristics of SB4 in field sections. (a) Interface of the Feixianguan Formation and the Jialingjiang Formation of the Qiaoting section; (b) fuchsia mud stone of the Feixianguan section; (c) dry crack of the Liuba section. 


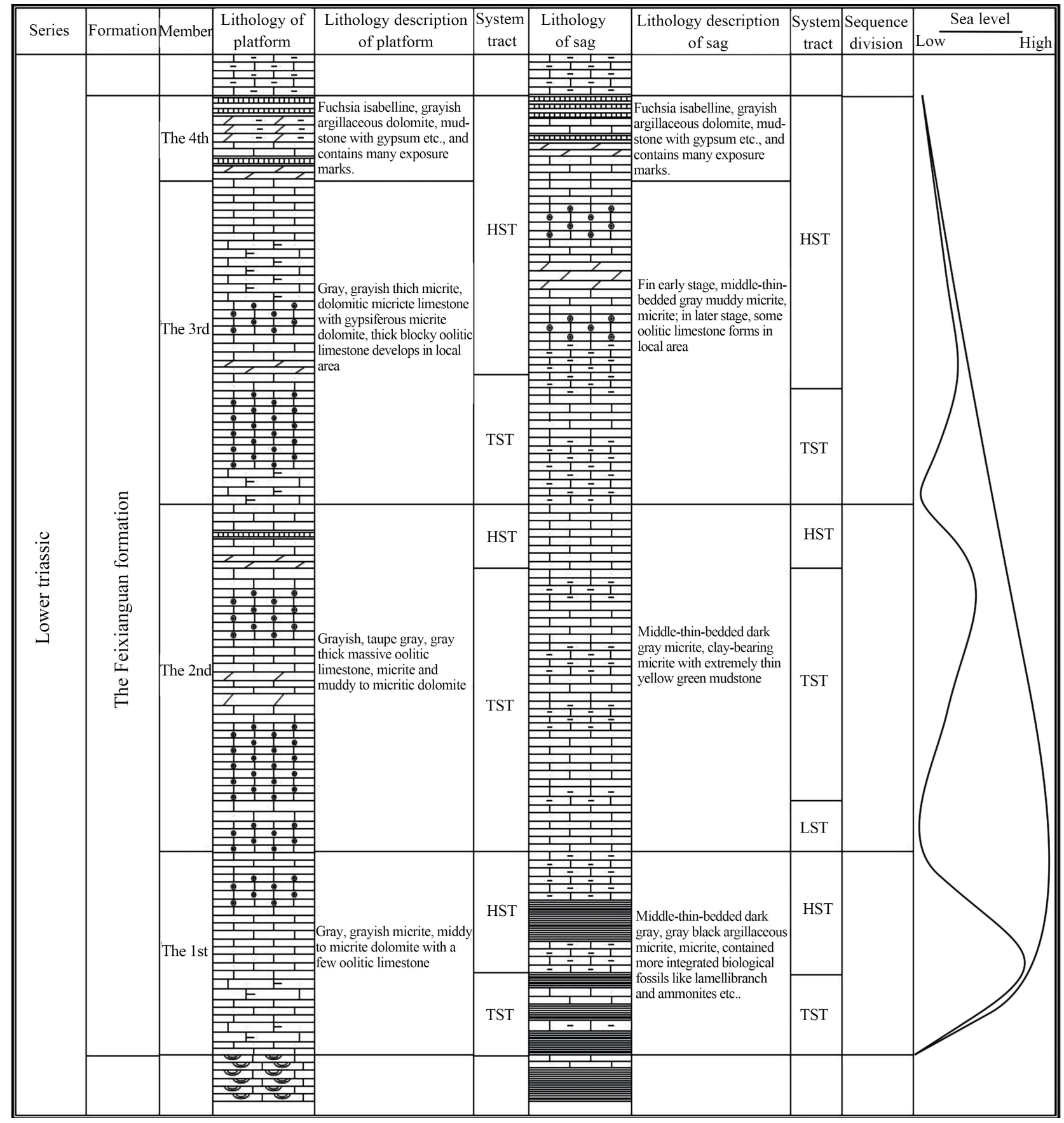

Figure 7. Sequence stratigraphic correlation column of the open platform and basin of the Feixianguan Formation in the Kaijiang-Liangping area.

Formation, northeast Sichuan, have been researched for many years. Improvements in exploration methods and research techniques have allowed researchers to determine that the Lower Triassic Feixianguan Formation has no hiatus between upper and lower members or differences in lithology; thus, the Feixianguan Formation inherited the lithofacies paleogeography of the Changing Formation [10] [11] [12]. The results of these previous studies combined with existing data were used in the present study to draw the following conclusion: The 
sedimentary model of the early Triassic Feixianguan period, northeast Sichuan, is a shallow sea carbonate platform. Based on the analysis of sedimentary facies characteristics in the Kaijiang-Liangping area according to drilling data, field outcrops, and laboratory analysis and tests, the study area was divided into evaporate platform facies and open platform facies. The latter includes subfacies as platform bank, or basin marginal bank, interbank, and platform basin (Figure 2, Table 2).

1) Evaporate platform facies

This facies is developed mainly in HST of the SQ3 sequence, corresponding to the member 4 of the Feixianguan Formation (Figure 6(a)). It formed in arid climate conditions, depositing a set of regional fuchsia mud stone (Figure 6(b)), gray argillaceous limestone, and micritic dolomite inter-bedded with gypsum. It includes horizontal bedding and obvious signs of exposure such as mud cracks and bird's-eye structures (Figure 6(c)).

2) Open platform facies zone

a) Platform bank (basin marginal bank)

This facies is developed in SQ1 and SQ2 sequences and TST of SQ3 sequence in the Feixianguan Formation, corresponding to the member 1, member 2 and member 3. Through wave action and screening of the coastal current, the major sediments include light-colored lenticular gray sparry oolitic limestone, clay-sparry bio-clastic (oolitic) limestone, and gravel (oolitic) limestone (Figure 8). Ripples, bimodal cross beddings, and small- to medium-sized scour structures are present. The thickness of the oolitic beach has high variation. The vertical distribution is not stable and is arranged as sheets or strips around the basin margin.

Table 2. Sedimentary features of the Feixianguan Formation in the Kaijiang-Liangping area.

\begin{tabular}{|c|c|c|c|c|c|}
\hline \multicolumn{2}{|c|}{ Types of sedimentary facies } & Lithology characteristics & Color & Sedimentary structure & Paleaobios \\
\hline \multirow{3}{*}{$\begin{array}{l}\text { Evaporat } \\
\text { platform }\end{array}$} & & Micrite, mudstone (shale) & & Thin-middle bedded, & \\
\hline & & gypsiferous dolomite, & Fuchsia, gray, taupe & horizontal bedding, dry crack, & sastropods, \\
\hline & & $\begin{array}{l}\text { gypsum, with a few grained } \\
\text { rock in local }\end{array}$ & gray and white & $\begin{array}{l}\text { birdeye structure and exposed } \\
\text { erosion }\end{array}$ & lamellibranchiate \\
\hline \multirow[t]{2}{*}{$\begin{array}{l}\text { Open } \\
\text { platform }\end{array}$} & Interbank & $\begin{array}{l}\text { Micrite, grainy micrite with } \\
\text { thin-layer micritic grain } \\
\text { limestone }\end{array}$ & Gray and dark gray & $\begin{array}{l}\text { Thin to middle layer, } \\
\text { horizontal bedding, ripple } \\
\text { bedding, burrow }\end{array}$ & $\begin{array}{l}\text { Gastropods } \\
\text { lamellibranchiate, } \\
\text { Ostracoda, alga }\end{array}$ \\
\hline & platform basin & Calcarenite, micrite, marl & $\begin{array}{l}\text { Grayish, dark gray } \\
\text { and taupe gray }\end{array}$ & $\begin{array}{l}\text { Middle to thin layer, horizontal } \\
\text { bedding, bioturbated structure }\end{array}$ & $\begin{array}{l}\text { Fossil fragments of } \\
\text { Gastropods, } \\
\text { lamellibranchiate } \\
\text { foraminifera }\end{array}$ \\
\hline
\end{tabular}



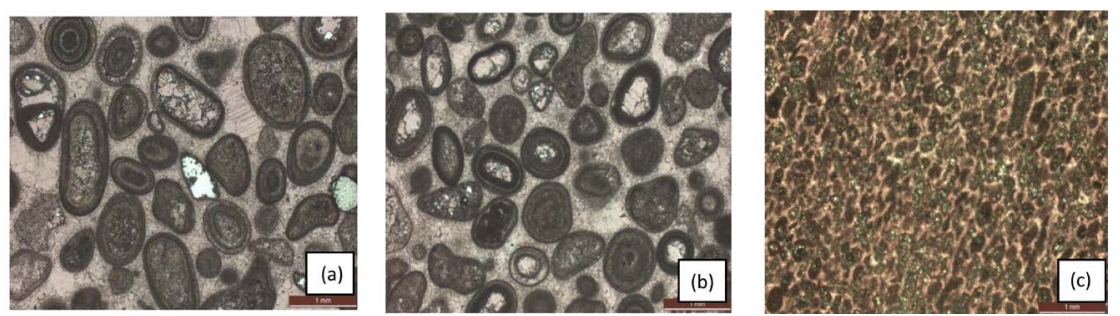

Figure 8. Sedimentary characteristics of platform bank of open platform in the Kaijiang-Liangping area, the member 2 of the Feixianguan Formation. (a) \& (b) Feixianguan section, light-colored lenticular gray sparry oolitic limestone; (c) Yudongliang section, plane-polarized light, $\times 2.5$.

\section{b) Interbank}

This facies is between platform banks with limited water circulation, dark-colored deposits, and fine-grained sediments in a wide distribution range. It exhibits a set of gray to dark gray thin-medium micrite and particles of micrite limestone interbedded with thin micritic particles of limestone. Horizontal beddings and sand grain beddings are present (Figure 9). Biology is active and includes include gastropods, lamellibranches, ostracods, and blue-green algae with burrows.

c) Platform basin

This facies lies inside the open platform. The depth of the water is from a few meters to hundreds of meters with normal water circulation; development is in the SQ1 or SQ2 sequence of the Feixianguan Formation. From the platform basin slope to the inner basin, remarkable differences in sedimentary characteristics are noted. In the platform basin slope zone, the water level is deeper, and deposits include a set of thin to medium dark gray micrite with slump breccia (Figure 10(a)) accompanied by a sliding deformation structure including gastropod and lamellibranch fossils. Within the platform basin, under adverse hydrodynamic conditions, a set of dark-colored, fine-grained dark gray micritic limestone, marlstone, and calcareous mudstone is present (Figure 10(b)). Horizontal beddings are developed, including oceanic plankton assemblages.

\subsection{Sequences and Sedimentary Facies of the Connecting-Well Section}

To determine the development law of sedimentary facies and the distribution of the oolitic beach in the Feixianguan Formation, a stratigraphic correlation of the third-order sequences in the Feixianguan Formation of the Kaijiang-Luanping area was made. Seven connecting-well sections covering the entire area (Figure 1) were selected to conduct a comparative study of the basin formation in parallel and perpendicular directions. Due to the paper length limitation, we selected only two representative sections for the present analysis: section 2 and section 7 .

Section 2 (Figure 11) is a connecting-well section of the Feixianguan Formation that crosses the basins of the Kaijiang-Liangping area. Among the facies of the entire section, a strong similarity is shown in which oolitic beaches are obviously controlled by facies. In the platform basin zone, the oolitic beaches are 
barely developed in wells Lg10 and Sm1. In the basin margins, wells Pg2 and Lg2 show several stages of basin marginal bank development with large thickness noted in the SQ2 sequence and TST of the SQ3 sequence, which is the member 3 of the Feixianguan Formation. Wells Lg11 and Lg9 in the open platform show partially developed oolitic beaches of various thickness. In the HST of SQ3 sequence, the platform is a homogenized evaporate platform, and the deposits include a typical set of regional purple calcareous mudstone, argillaceous dolomite, and micrite interbedding with gypsum.

Section 7 (Figure 12) is a connecting-well section of the Feixianguan Formation running parallel with the margin of the Kaijiang-Liangping basin and extending through Jiulongshan. In the Longgang and Longhuichang areas, the changes in sedimentary facies are essentially revealed in the basin margins. Wells L4 and Lh3 are located at Jiulongshan and the Longhuichang area respectively. The thickness of the Feixianguan Formation is relatively small, and oolitic beaches are deposited in the TST of wells L4 SQ3 sequence and well Lh3 SQ1, SQ2, and SQ3 sequences, which indicate shallow water with strong hydrodynamic power in both places in the Feixianguan period. These represent two high points in the shallow water region. Wells $\mathrm{Yb} 4$ and $\mathrm{Lg} 10$ are located in the basin near the Longgang area. The thickness of the Feixianguan Formation is large, which suggests deep water is with week hydrodynamic power. Little or no particle deposition is noted in the Feixianguan period.

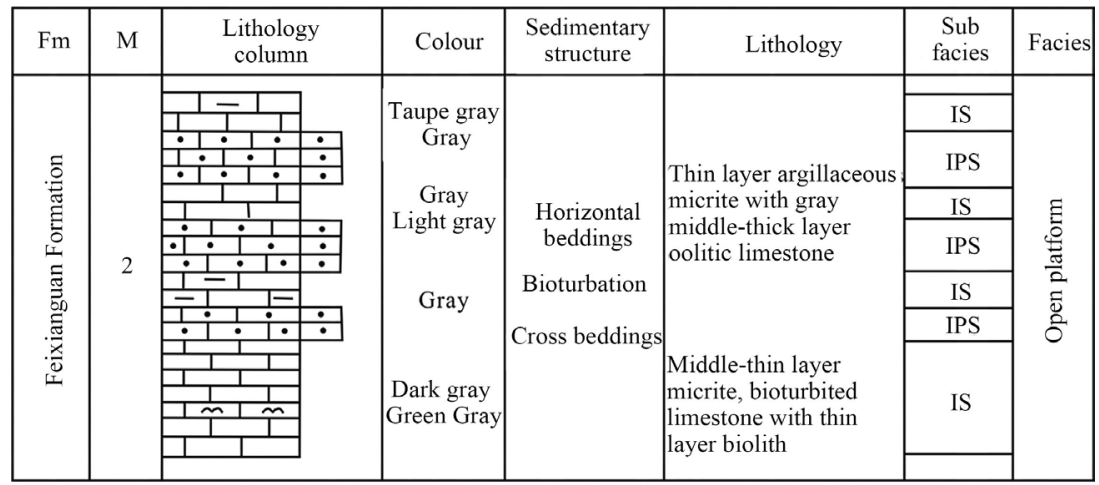

Fm: Formation; M: Member; IPS: Intra platform shoal; IS: Inter shoal

Figure 9. Sedimentary characteristics of interbanks of open platform in the Kaijiang-Liangping area, the member 2 of the Feixianguan Formation, Yudongliang section.
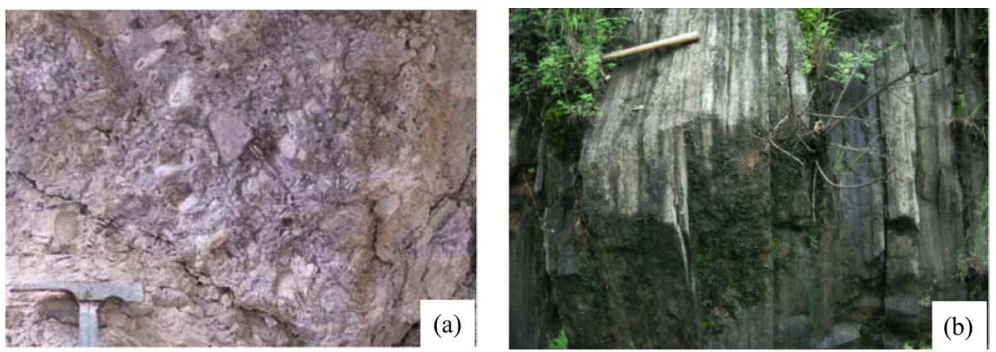

Figure 10. Sedimentary characteristics of the platform basin of the Kaijiang-Liangping area. (a) Slump breccia, the second member of the Feixianguan Formation, Xuanhan section; (b) Gray-green argillaceous micrite and mudstone of $\mathrm{T}_{1} \mathrm{f}^{1}$. 


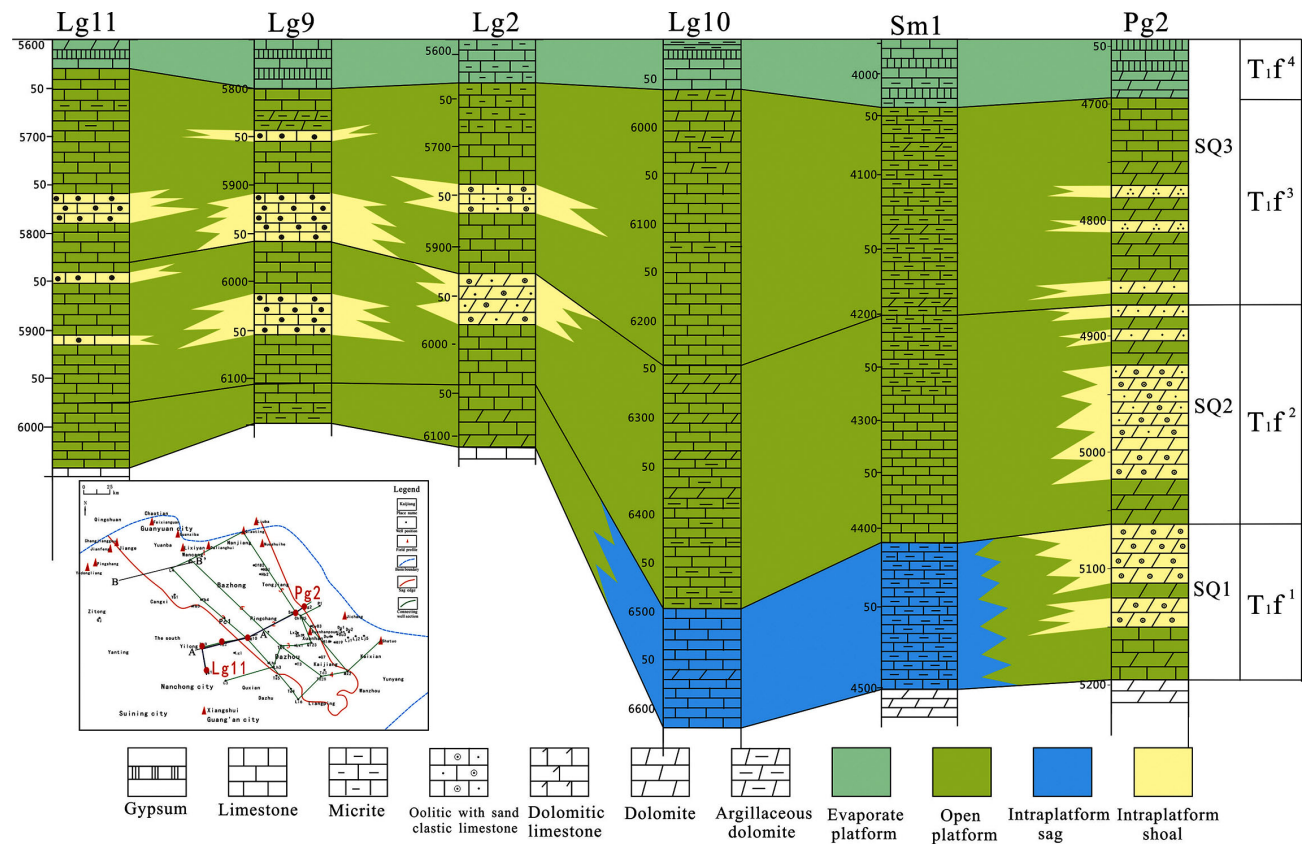

Figure 11. Sequences and sedimentary facies correlation of Lg11-Pg2 connecting-well section.

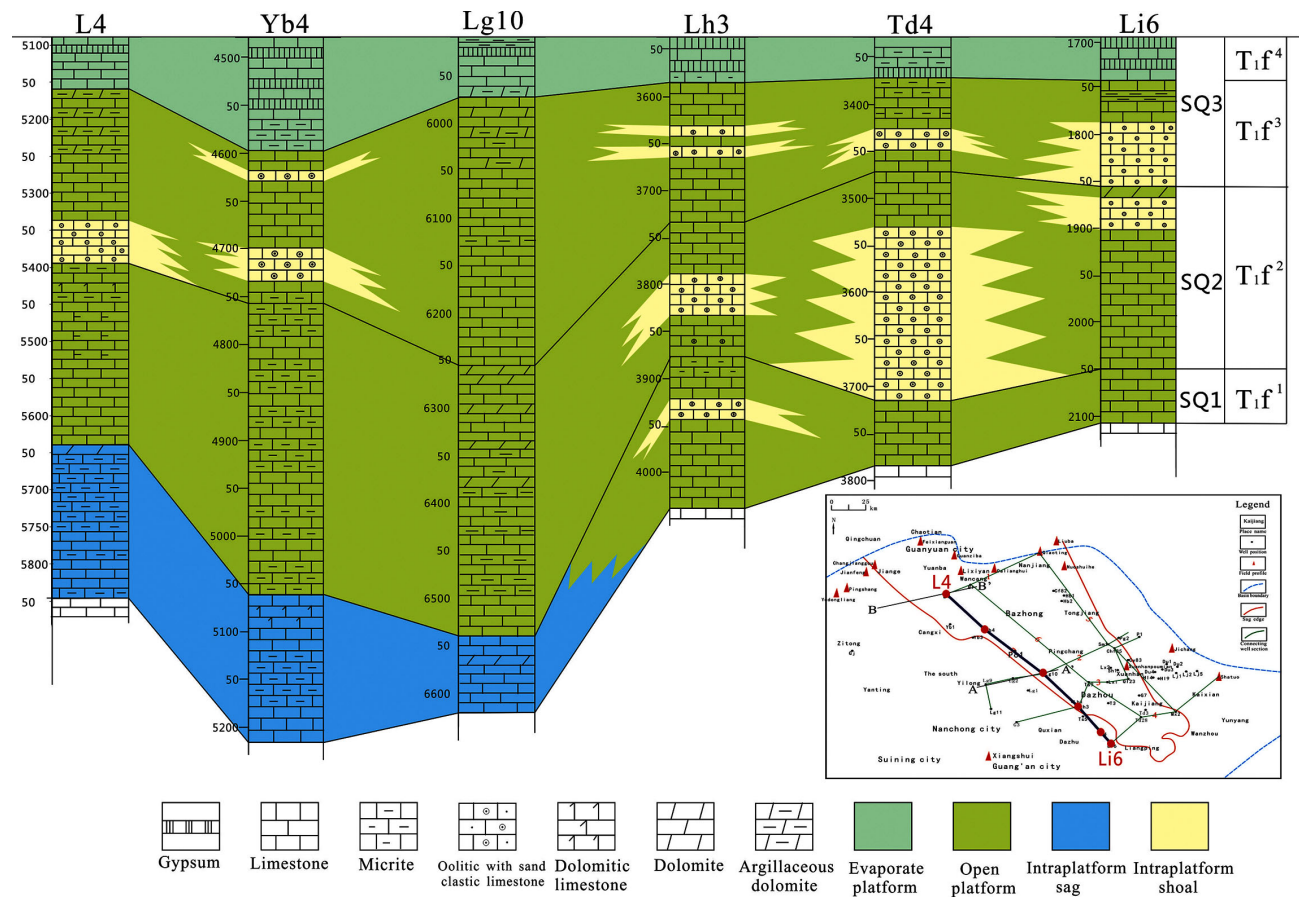

Figure 12. Sequences and sedimentary facies correlation of L4-Li6 connecting-well section.

Comparisons were conducted for the sequences and sedimentary facies of the connecting-well members parallel and perpendicular to the Kaijiang-Luanping basin. The comparison characteristics of the sequences in the open platform and platform basin are obvious in the Feixianguan period such that oolitic beaches are distributed mainly around basin margin and that their development was controlled by sedimentary facies. Moreover, the basin margin advanced far away 
to the inner basin in Longhui and Jiulongshan. In the regression process of the late Feixianguan period, it could be easily developed into a sedimentary area with strong hydrodynamic conditions, which was favorable for the deposition and formation of grainstones. These areas show potential for further exploration.

\subsection{Sedimentary Evolution and Models}

According to the characteristics of the sedimentary facies in the study area, combined with the analysis of connecting-well stratigraphic sequence correlation, planar graphs of sedimentary facies evolution were mapped.

It is widely accepted that the sedimentary framework of the early Feixianguan period (SQ1 depositional period) was essentially inherited from the Changxing period. During the late Changxing period, rapid mass transgression created the facies of Kaijiang-Liangping area. Mainly platform basin, these facies indicate a sedimentary period with deep water and wide coverage where the main deposit was marl. Entering the Feixianguan period, the transgression reached the maximum flooding surfaces quickly and began to decline. At that time, a small number of oolitic beaches were deposited in the open platform with strong hydrodynamic power. These oolitic beaches were distributed mainly in the north area of Guangyuan along eastern basin margins in Puguang, Tieshanpo, Dukouhe, and Luojiazhai and western basin margins in Longgang, Tieshan, Longhuichang, and Liangping. The oolitic beaches in the north area are thicker than those in the south area, which illustrates that an early regressive stage with strong hydrodynamic power occurred in the north area before that in the south area. The sedimentary framework changed after this stage, although the overall framework was similar to that in the Changxing period (Figure 13).

The sedimentary framework of the SQ2 sequence was essentially inherited from the SQ1 sequence. Although the depositional water became shallow that than in the former period, it was a vital sedimentary period of the Feixianguan Formation. During this period, rapid sedimentation at the east margin and the Longgang area of the west margin caused the margins to move toward the inner basin with a platform margin propulsion distance of up to $40 \mathrm{~km}$ [13]. Thus, the scope of the basin reduced, and larger thicknesses of oolitic banks and bars formed on both margins and were concentrated mainly along the $\mathrm{Pu}$ guang-Luojiazhai and Yuanba-Longgang-Liangping area. The cumulative oolitic beach thickness at the platform margin could have reach 80 - $90 \mathrm{~m}$ with a maximum single layer thickness of $40 \mathrm{~m}$ [14]. The oolitic beaches deposited during this period could have been dolomitized to form good reservoirs through later diagenesis modification; the main gas-producing zone of Longgang1, Longgang2, and Puguang2 wells are the oolitic beaches reservoirs formed during this period. In summary, the shallow sea water caused the margins to advance toward the inner basin. The area of the basin was reduced, and the entire sedimentary framework underwent slight changes (Figure 14). 


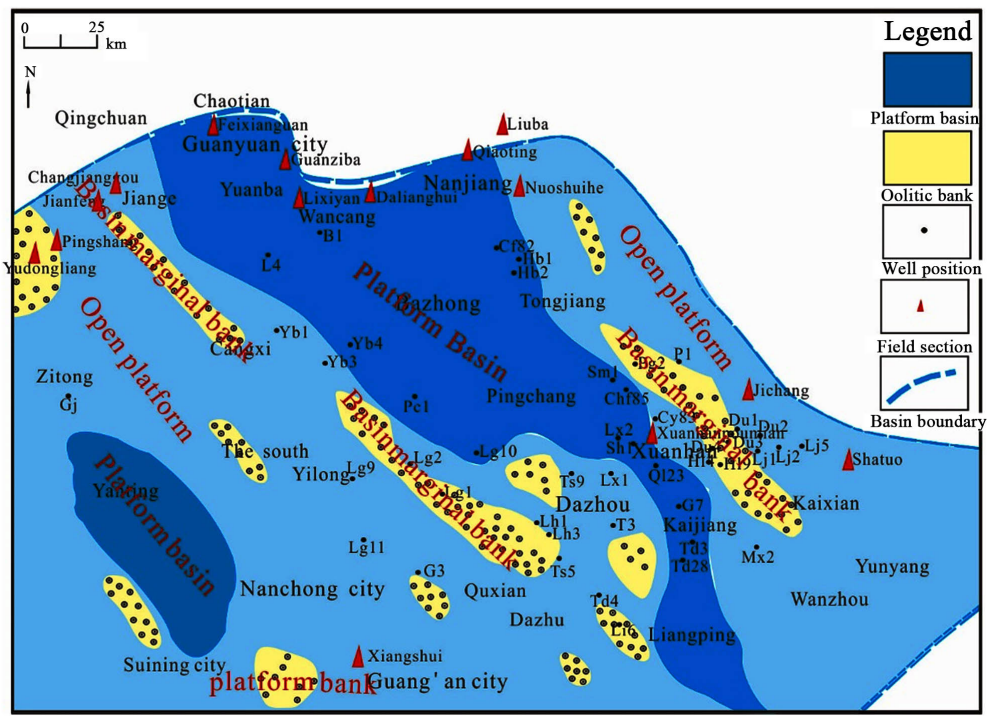

Figure 13. Sedimentary facies of the SQ1 $\left(\mathrm{T}_{1} \mathrm{f}^{1}\right)$ of Kaijiang-Liangping basin.

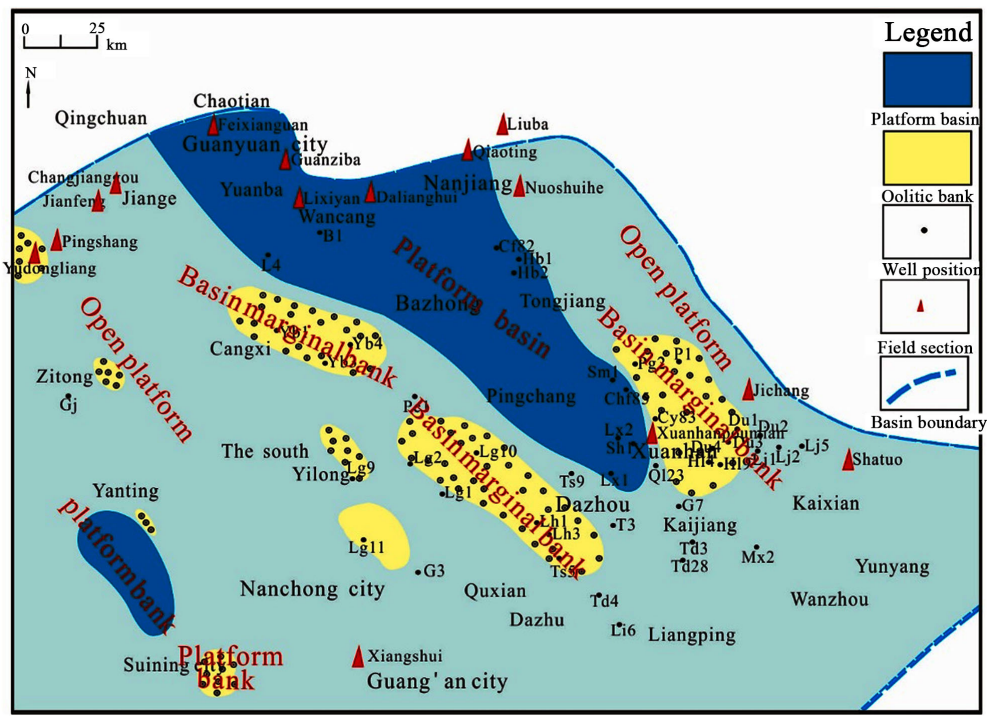

Figure 14. Sedimentary facies of the SQ2 $\left(\mathrm{T}_{1} \mathrm{f}^{2}\right)$ of Kaijiang-Liangping basin.

Entering the TST period of the SQ3 sequence, the sea water was shallow, and sedimentation was enhanced. The Kaijiang-Liangping basin was filled quickly until it finally disappeared, and the entire study area evolved into the open platform facies, with oolitic beaches deposited extensively in the platform and the basin area. During this period, the sedimentary framework changed completely. In addition to the filling of the Kaijiang-Liangping basin, the Feixianguan Formation was essentially filled at the regional scale. In HST of the SQ3 sequence, the entire Kaijiang-Liangping area evolved into a homogenized evaporate platform facies and developed a set of regional purple mud shale and marl with gypsum layers (Figure 15, Figure 16).

According to the evolution process of plane facies of the Kaijiang-Liangping area, we established a sedimentary facies model of the study area (Figure 17), 
showing the complete sedimentary filling process of the Feixianguan Formation.

\section{Prediction of Favorable Exploration Areas in the Kaijiang-Liangping Basin}

Before constructing the sedimentary model of the Feixianguan Formation of Kaijiang-Liangping basin, the characteristics of the margins needed to be identified. The Kaijiang-Liangping basin margins show faulting at the east and overlapping at the west [5] [15]. The east faulting margin is steep, and the sedimentary characteristics show little change as sea level changes. The positions of favorable reservoir development were stable and distributed mainly along the basin margin of Tieshanpo-Puguang-Dukouhe-Luojiazhai-Yun'anchang. Past the

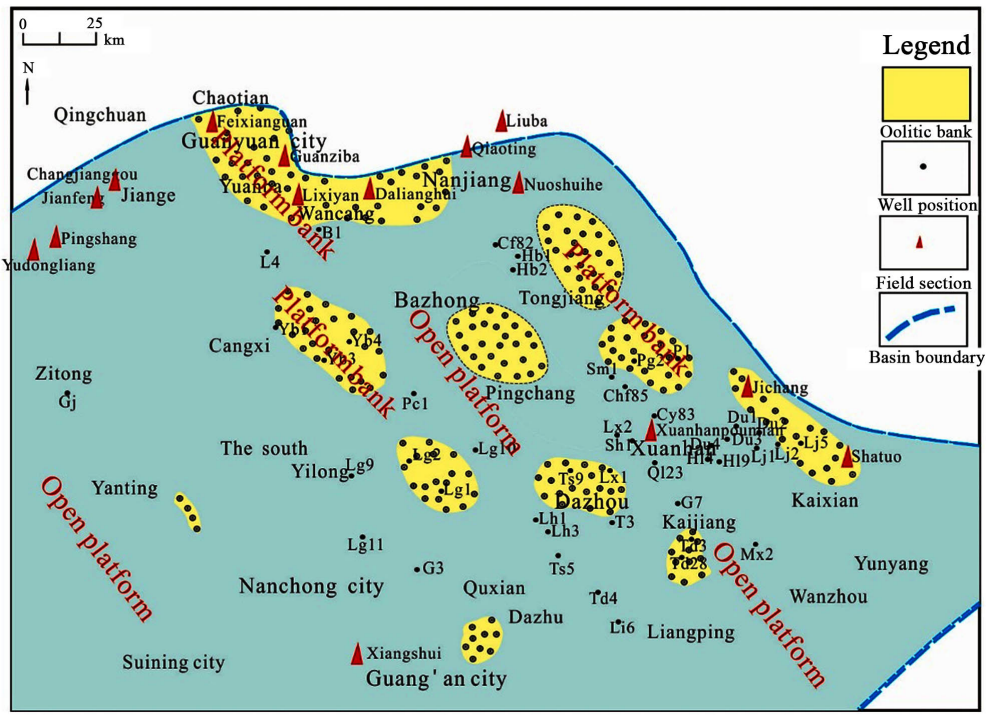

Figure 15. Sedimentary facies of the TST of SQ3 $\left(\mathrm{T}_{1} \mathrm{f}^{3}\right)$ of Kaijiang-Liangping basin.

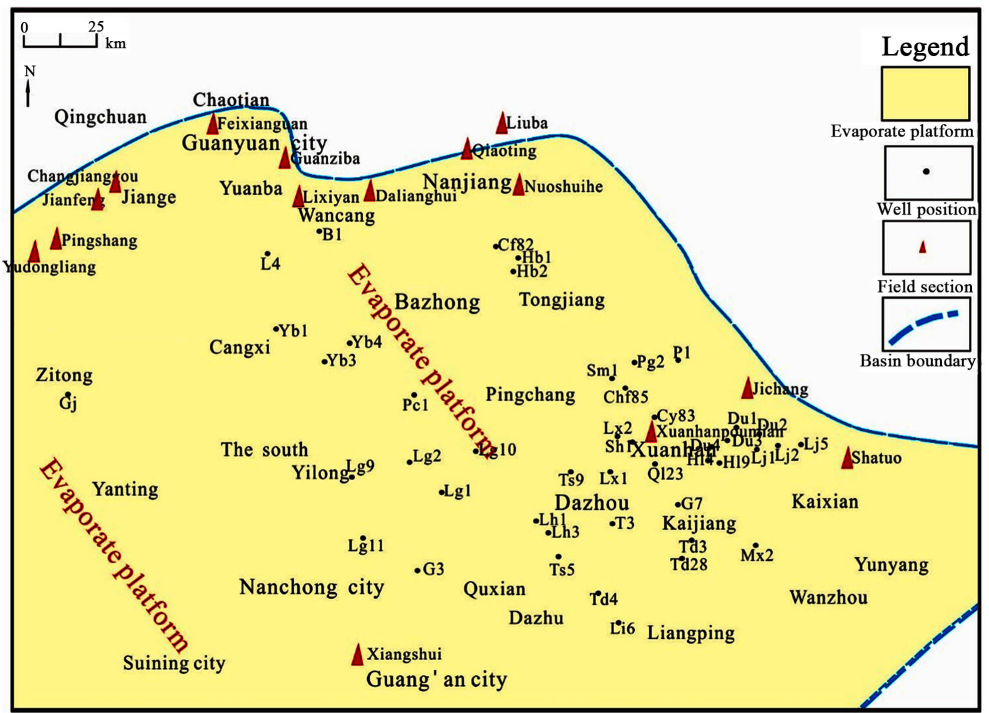

Figure 16. Sedimentary facies of the HST of SQ3 $\left(\mathrm{T}_{1} \mathrm{f}^{4}\right)$ of Kaijiang-Liangping basin. 


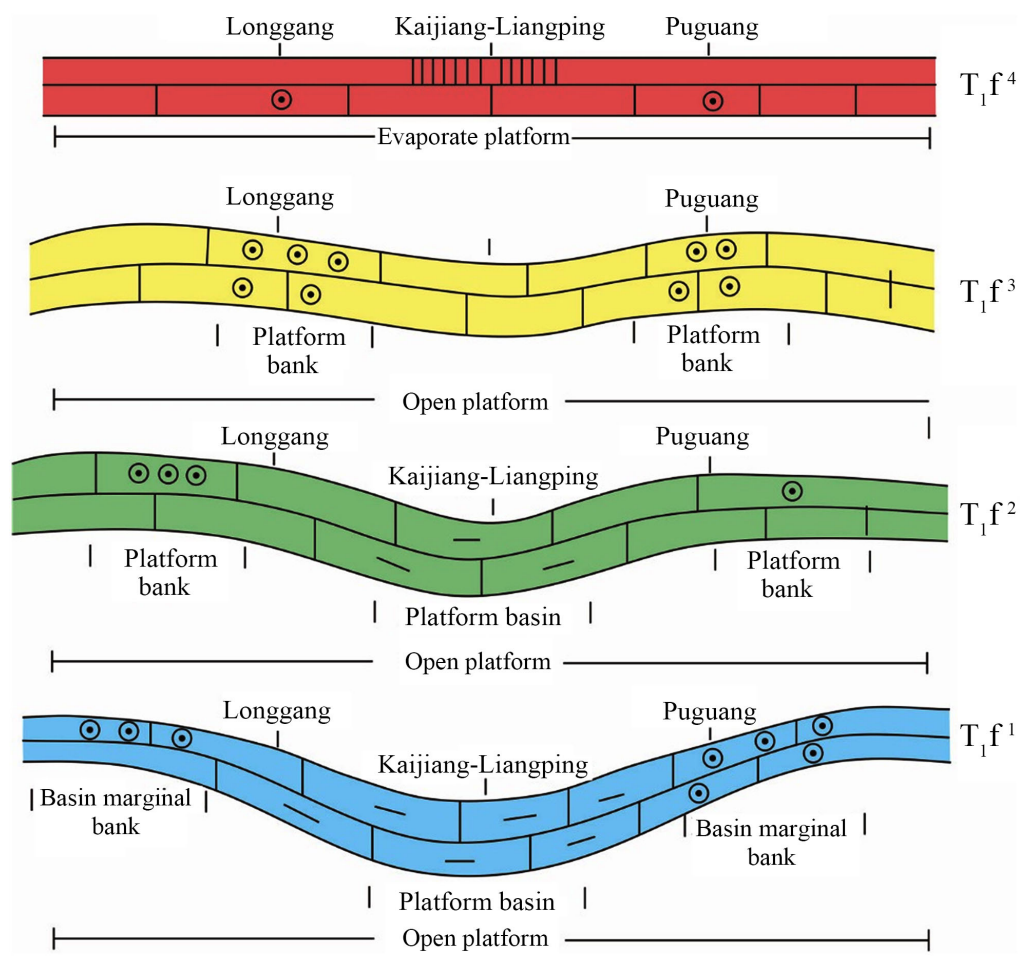

Figure 17. Evolution model of the Feixianguan Formation in the Kaijiang-Liangping area.

slope toward the inner basin, the water deepened suddenly, where was unfavorable for sedimentation of oolitic banks and development of good reservoirs. Therefore, the favorable exploration areas are situated along the east margin. At the west margin, except for areas along the margin such as Yuanba-Longgang-Shuangjiaba, the earlier sediments at Jiulongshan and the Longhui area advanced faster to the basin. Strong hydrodynamic conditions formed during the regression allowed the sedimentation of oolitic beaches to form favorable reservoirs, which may become a potential favorable exploration zone. Because the Kaijiang-Liangping area has low exploration potential and severely lacks drilling and logging data, the $2 \mathrm{D}$ and $3 \mathrm{D}$ seismic data of the study area were used to analyze the characteristics of the seismic profiles at the west margin. On the basis of the sequences and sedimentary facies data, two development models of oolitic beach are offered [16].

1) Aggradation model

The aggradation model is developed mainly in the Longgang area. Faults are not developed because of weak tectonic deformation; the seismic reflection is very clear. From the reflection characteristics of seismic member across wells such as Longgang 9, Longgang 2, and Longgang 10 (Figure 1, AA'; Figure 18(a)), the characteristics of the bottom interface reflection axes of the member 2 and member 3 of the Feixianguan Formation are essentially correlated with those of the member 1 of the Feixianguan Formation. Both become steeper suddenly at the basin margin and extend to the inner basin. At the basin margin, 
the characteristic the bottom interface reflection axis of the member 1 of the Feixianguan Formation is moderately strong, continuous, and clear. The bottom interface reflection of the member 2 of the Feixianguan Formation is weak, and the bottom interface reflection axis of the member 3 of the Feixianguan Formation shows "bright spot" reflection characteristics (Zhu et al., 2000). The reflection characteristics of the seismic section of the Feixianguan Formation in the Longgang area indicate that oolitic beaches develop mainly at the basin margin and in the member 3 of the Feixianguan Formation. Logging data shows that from the SQ1 sequence to TST of the SQ3 sequence, well Longgang 9 includes micrite, argillaceous limestone, and sand chip limestone mixed with a small number of oolitic beaches. Well Longgang 2 at the basin margin includes significantly thicker oolitic beach layers. Well Longgang 10 in the basin slope shows dark gray micrite or green mud limestone with almost no oolitic beach development. The Longgang model was established according to the sedimentary characteristics of the Longgang area (Figure 18(b)).

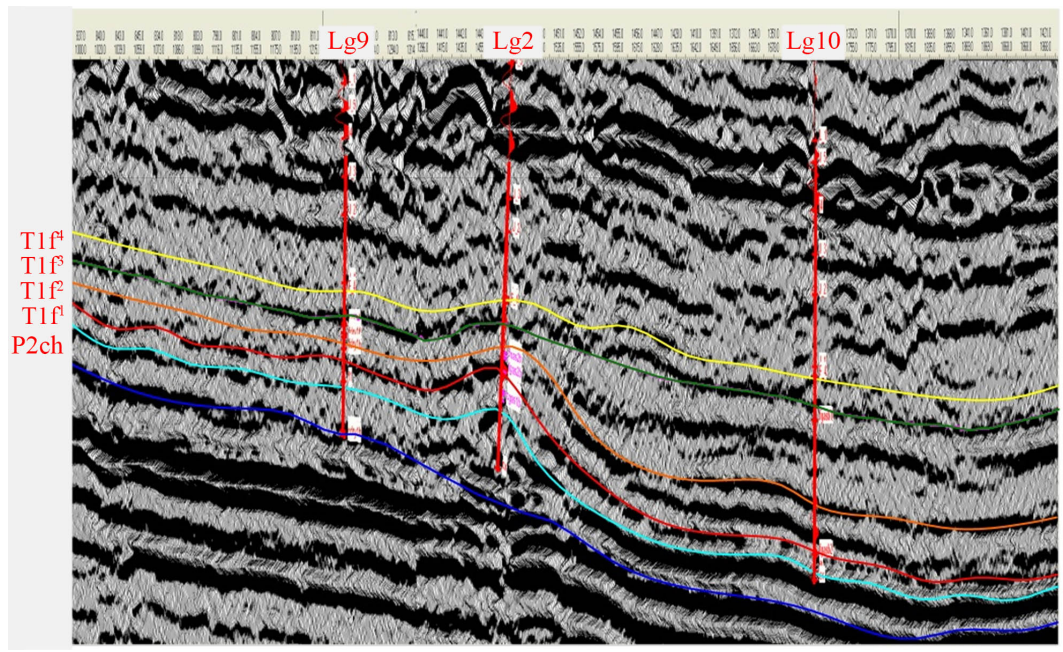

(a)

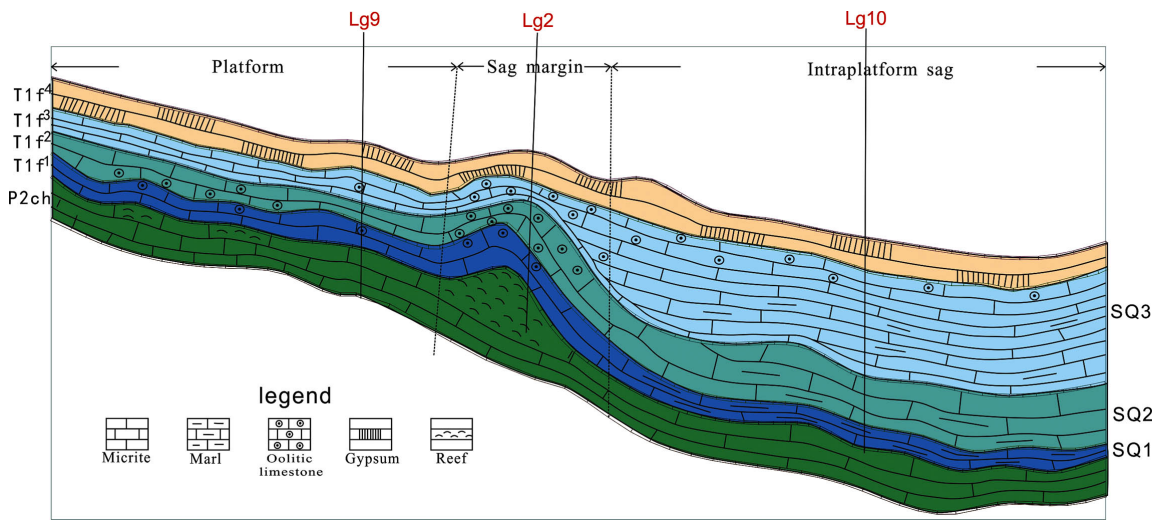

(b)

Figure 18. Seismic characteristics and aggradation model graph of the Longgang area in the Kaijiang-Liangping basin. (a) AA' seismic section, (b) Aggradation model of Longgang area. 


\section{2) Progradation model}

This model is developed mainly in the Jiulongshan and Longhui areas. The seismic reflection characteristics of the Longhui area are not obvious owing to the complicated construction conditions. The seismic reflection characteristics of the Jiulongshan area are clearer and were thus analyzed in this study. The reflection characteristics of the seismic section of wells such as Jianmen 1 to Long 4 show that the member 4 of the Feixianguan Formation is flatter; well Long 16 is in close proximity to well Long 4 (Figure 1, BB'; Figure 19(a)). The bottom interface reflection axis of the member 1 of the Feixianguan Formation bends slowly from the margin to the inner area after a certain distance, then extends closely to the bottom interface reflection axis of the Changxing Formation. The bottom interface reflection axis of the member 2 of the Feixianguan Formation advances slowly from the margin to the inner area after a certain distance, then bends downward to the bottom interface reflection axis of the member 1 of the Feixianguan Formation. It then extends to the basin center, where bright spot reflection is shown on the propelling extension axis. The bottom interface reflection axis of the member 3 of the Feixianguan Formation is almost an extended parallel from the margin to the inner area. The reflection characteristics of the Jiulongshan seismic section indicate that oolitic beaches are developed mainly at the basin margin and near the top interface of the member lof the Feixianguan Formation. Well logging data shows that well Jianmen 1 includes mainly deposits of argillaceous limestone and thick layers of limestone, with thin layers of oolitic limestone occurring only in HST of the SQ1 sequence and TST of the SQ2 sequence. At the location of well Longgang 16, the oolitic limestone is developed mainly in the SQ1 sequence and TST of the SQ3 sequence. The development of oolitic beach reservoirs migrates from the margin to the inner basin in the entire area. The migration distance from the margin to the inner basin in Jiulongshan is approximately $34 \mathrm{~km}$; the local area maximum is $40 \mathrm{~km}$. The Jiulongshan model was established according to the sedimentary characteristics of the Feixianguan Formation in the Jiulongshan area (Figure 19(b)).

In summary, the two types of oolitic beach development models revealed that favorable exploration zones of the Feixianguan Formation in the Longgang area occur mainly in the margin zone of oolitic beaches development. The favorable exploration zones in Jiulongshan and Longhui advanced further to the inner basin.

\section{Conclusions}

1) According to the characteristics of sequence interfaces, the Feixianguan Formation of the Kaijiang-Liangping area can be divided into three third-order sequences: SQ1, SQ2, and SQ3. SQ1 and SQ2 correspond to the member 1 and the member 2, respectively, and SQ3 corresponds to the member 3 and the member 4. In the platform, both TST and HST are developed in the SQ1, SQ2, and SQ3 sequences. However, in the basin, TST and HST are developed in the SQ1 and SQ3 sequences, and LST, TST, and HST are developed in the SQ2 sequence. 


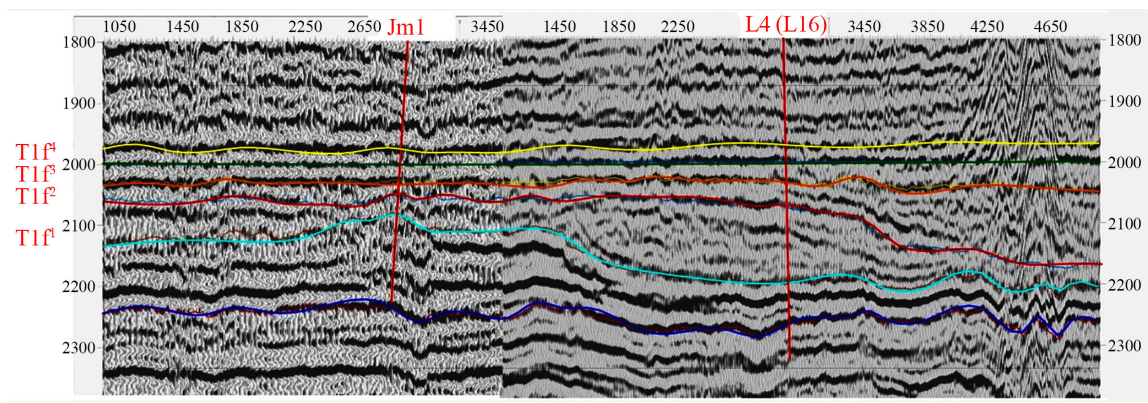

(a)

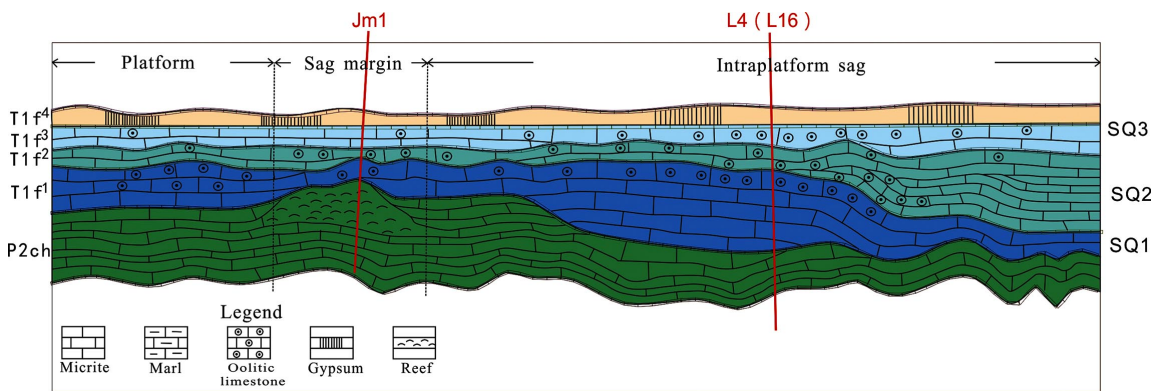

(b)

Figure 19. Seismic characteristics and aggradation model graph of the Jiulongshan area in the Kaijiang-Liangping basin. (a) BB' seismic section, (b) Progradation model of the Jiulongshan area.

2) The sedimentary facies of the Feixianguan Formation in the Kaijiang-Liangping area can be divided into open platform facies and evaporate plateform facies. The open platform includes three subfacies: platform bank, interbank, and platform basin. During the first and second periods of the Feixianguan Formation, the study area was mainly platform bank, interbank, and platform basin subfacies. In the third period, the basin was filled and leveled, and the major facies were platform bank and interbank subfacies. In the fourth period, the entire area evolved into evaporated platform facies.

3) On the basis of the seismic reflection characteristics and the sedimentary facies, two types of development models for oolitic beaches at the west margin of the Kaijiang-Liangping basin are proposed. The aggradation model shows development mainly in the Longgang area. Oolitic beaches are developed in the SQ1 and SQ2 sequences and in TST of the SQ3 sequence of the basin margin, which are favorable exploration areas. The progradation model shows development in the Jiulongshan and Longhui areas with oolitic reservoirs occurring mainly in HST of SQ1 and TST of SQ3. With the progradation to the inner basin, the basin margin advanced gradually, the oolitic banks migrated into the basin along with the progradation.

\section{Acknowledgements}

The support from Science Foundation of China University of Petroleum-Beijing at Karamay (No. RCYJ2016B-01-003) is highly appreciated, and supported by National Science and Technology Major Project (2017ZX05063002-006). 


\section{Conflicts of Interest}

The authors declare no conflicts of interest regarding the publication of this paper.

\section{References}

[1] Wang, Y.G., Wen, Y.C., Zhang, F., Yang, Y. and Zhang, J. (1998) Distribution Law of Reefs of the Upper Permian ChangXing Formation, East Sichuan. Natural Gas Industry, 6, 25-30+7-8.

[2] Yang, Y. and Wen, Y.C. (2002) Control of KaiJiang-LiangPing Ocean Trough for the Oolite Beaches' Distribution of Feixianguan Formation in Northeast Sichuan Basin. Natural Gas Industry, No. S1, 30-32+10.

[3] Wei, G.Q., Chen, G.S., Yang, W., Yang, Y., Jia, D., Zhang, L., Xiao, A.C., Chen, H.L., Wu, S.X., Jin, H. and Shen, J.H. (2006) Preliminary Study of the Boundaries Characteristics of Kaijiang-LiangPing Trough in Northern Sichuan Basin and Its Characteristics. Oil \& Gas Geology, 27, 99-105.

[4] Ma, Y.S., Mou, C.L., Tan, Q.Y. and Yu, Q. (2006) A Discussion on Kaijiang-Liangping Ocean Trough. Oil \& Gas Geology, 27, 326-331.

[5] Zhou, Q., Zhuo, S.G., Wei, G.Q. and Zhang, L. (2008) Sequence Stratigraphic Analysis of the Lower Triassic Feixianguan Formation, Northern Sichuan Basin. Journal of Stratigraphy, No. 4, 406-410.

[6] Qiao, Z.F., Li, G.R., Long, S.X., Jiang, Z.Z., Hu, W.Y. and Li, W.M. (2010) Characteristics and Evolution Model of Sequence Stratigraphy of Feixianguan Formation in the Northeast of Sichuan Basin. Acta Sedimentologica Sinica, 28, 462-470.

[7] Tan, X.F., Lie, J., He, J.P. and Peng, P. (2012) Sequence Lithofacies Paleogeographic Characteristics of Feixianguan Formation in Southern Area of Kaijiang-Liangping Ocean Trough. Geology in China, 39, 612-622.

[8] Du, Y.S., Yin, H. and Wang, Z.P. (1997) The Basin's Framework and Tectonic Evolution of QinLing Orogenic Belt during the Late Caledonian-Early Hercynian. Earth Science-Journal of China University of Geosciences, 22, 401-405.

[9] Zhu, X.M. (2000) Sequence Stratigraphy. China University of Petroleum Press, Qingdao, 42-43.

[10] Wang, Y.G., Hong, H.T., Xia, M.L., Fan, Y. and Wen, Y.C. (2008) Exploration of Reef and Beach Reservoirs of Permian and Triassic Surrounding Troughs in Sichuan Basin. Natural Gas Industry, 1, 22-27+161-162.

[11] Zhang, Y.C., Yang, A.G., Mei, Y., Deng, Q.H. and Chen, H. (2009) Seismic Prediction of Extensive Kaijiang-Liangping Trough and Favorable Facies Belts. Natural Gas Industry, No. 10, 28-30+136-137.

[12] Wen, L., Zhang, Q., Yang, Y., Liu, H.Y., Che, Q., Liu, W., Zeng, Y.X., Wei, X.W., $\mathrm{Xu}$, L., Liu, M. and Liu, L. (2012) Distribution Controlling Factors of Reef and Beach and Favorable Exploring Areas in the Changxing-Feixianguan Formations in the Sichuan Basin. Natural Gas Industry, 1, 39-44+120-121.

[13] Zhang, J.Y., Zhou, J.G., Hao, Y., Wang, X.F., Lü, Y.Z., Zhang, D.Z., Xu, M.R., Zhang, R.H., Gu, M.F. and Zhang, J.Y. (2011) A Sedimentary Model of Changxing and Feixianguan Formations around Kaijiang-Liangping Trough in Sichuan Basin. Marine Origin Petroleum Geology, No. 3, 45-54.

[14] Zhou, H., Zhao, Z.J., Liu, Y., Luo, Z., Zhang, B.M., Liu, Y., Zhang, X.Y., Jiang, Q.C., Li, Q.F., Gao, Z.Y., Yang, Y. and Liu, Y.H. (2012) Sequence Lithofacies Paleogeo- 
graphy and Favorable Exploration Zones of Indian Age of the Lower Triassic in Sichuan Basin and Adjacent Areas, China. Acta Petrol Sinica, 33, 52-63.

[15] Wei, G.Q., Chen, G.S., Yang, W., Yang, Y., Hu, M.Y., Zhang, L., Wu, S.X., Jin, H. and Shen, J.H. (2004) Sedimentary System of Platformal Trough of Feixianguan Formation of Lower Triassic in Northern Sichuan Basin and Its Evolution. Acta Sedimentologica Sinica, 22, 254-260.

[16] Li, Y.F., Liu, S. and Zeng, X. (2005) Seismic Response Features and Prediction of Oolitic Beach Reservoirs of Feixianguan Formation in East Sichuan Basin. Geophysical Prospecting for Petroleum, 44, 236-239. 